\title{
INFLAMMATORY BOWEL DISEASE IN NEWBORN PIGS: THE ROLE OF FORMULA-FEEDING AND PREMATURE BIRTH
}

\author{
Per T Sangild, Charlotte R Bjørnvad, Yvette M. Petersen, Douglas G Burrin, Jan Elnif \\ and Mette Schmidt
}

Animal Nutrition and Reproduction, Royal Veterinary and Agricultural University, Frederiksberg C, DK-1870, Denmark

Various forms of inflammatory bowel disease are commonly observed among farm animals in the immediate postnatal period. At this critical time, only mild detrimental effects on body metabolism and fluid balance are tolerated and digestive disturbances contribute significantly to a high neonatal mortality among farm animals. We hypothesized that 1) colostrum contains factors that protect against intestinal disease, and 2) intestinal disease is particularly prevalent in pigs born prematurely. The rationale for these hypotheses was derived from the observation that preterm birth coupled with formula-feeding is associated with markedly increased incidence of necrotizing enterocolitis (NEC) in humans. This intestinal disease is the most common and most severe intestinal disease in premature infants. Premature pigs were delivered by elective caesarean section ( $105 \mathrm{~d}$ gestation, term $=115$ d), kept in infant incubators, and fed either sow's colostrum (COLOSTRUM, n=13) or milk-replacer (FORMULA, $\mathrm{n}=14$ ) every $3 \mathrm{~h}$. An additional 12 pigs were delivered at term and reared in a similar manner. All pigs were killed at $24 \mathrm{~h}$ after birth for clinical evaluations and tissue collection. Intestinal structure and function was measured in the distal region of the gut.

Table. Intestinal structure and function in premature pigs fed colostrum or formula

\begin{tabular}{lcc}
\hline & Colostrum & Formula \\
\hline Disease incidence (\%) & 0 & $57^{*}$ \\
Blood acidity (pH) & $7.43 \pm 0.04$ & $7.24 \pm 0.03^{*}$ \\
Intestinal mucosa (\%) & $75.6 \pm 1.1$ & $65.0 \pm 3.5^{*}$ \\
Villus height (:m) & $556 \pm 37$ & $263 \pm 37^{*}$ \\
Maltase activity (U/g) & $6.18 \pm 0.89$ & $1.31 \pm 0.28^{*}$ \\
Lactase activity (U/g) & $19.6 \pm 2.8$ & $10.2 \pm 2.2^{*}$ \\
Aminopeptidase A (U/g) & $4.22 \pm 0.28$ & $2.73 \pm 0.34^{*}$ \\
Glucose absorption (U/g) & $1.02 \pm 0.09$ & $0.59 \pm 0.07^{*}$ \\
\hline
\end{tabular}

Typical symptoms of severe NEC (gas-distended bowel, watery and bloody diarrhoea, extensive necrosis of gastric and intestinal mucosa, blood acidosis) were observed in 8 of the 14 formula-fed premature pigs $(57 \%$, Table). No NEC symptoms were evident in premature pigs fed colostrum $(0 / 13$, Table), or in term pigs fed formula $(0 / 6)$ or colostrum $(0 / 6)$. The distal small intestine of premature formula-fed pigs also showed marked decreases in mucosal mass, villus height, activity of mucosal enzymes (maltase, lactase, ApA) and glucose absorption (*, $\mathrm{P}<0.05$ relative to COLOSTRUM). We conclude that caesarean-delivered premature pigs fed formula show a high incidence of a severe intestinal disease in the immediate neonatal period. The clinical signs of this disease are similar to those of necrotizing enterocolitis (NEC) in premature infants. The premature formula-fed pig can be used as a model to investigate the factors in colostrum that provide protection against intestinal disease in both normal and premature newborns. 